\title{
Consultants and clinical team management
}

\author{
John Oldham
}

Over the past 10 years or so, management has gained greater significance as a career choice for doctors. They see enormous opportunities to improve clinical care by having a greater say in setting the agenda of their organisation and directing the deployment of the local healthcare budget. While doctors have always enjoyed a position of major influence over the direction of the health service, real opportunities to operate as managers only became available in the mid 1980s with the implementation of the Griffiths Report (Department of Health and Social Security, 1983). Although mental illness hospitals had Medical Superintendents until the 1970s, professional hierarchies and consensus decision making, combined with incremental planning and an administrative culture, resulted in few opportunities for meaningful involvement of doctors in management.

Today the situation is quite different. The 1991 NHS Reforms (Department of Health, 1989) introduced business management and emphasised the need for pro-active management as the vehicle for delivering cost effectiveness, service reorientation, quality assurance and evidence based practice.

Combined with a rapid development of new technology and a greater emphasis on consumerism, the health service is experiencing a revolution. All consultants are managers and as leaders in the service they need to develop their management potential, whether it is to lead the clinical team or manage a range of services. This paper explores some of the issues raised for consultants by this challenge and suggests how they might prepare for management responsibilities. Consultants intending to tread the management path need to do far more than attend a few courses to acquire new skills and knowledge. It is essential that their programme of preparation involves gaining a detailed understanding of the wider political and economic environment in which the health service operates. The adoption of an appropriate leadership style is also vital and the dilemmas posed by the different value sets of doctors and managers must be faced and resolved. Formal management training is an important component of the preparation process.

\section{The clinical team}

The clinical team ranges from the consultant-led firm of doctors in the hospital to the multidisciplinary team delivering a comprehensive service; e.g. a clinical directorate delivering a comprehensive child mental health service across a whole health district. Between the two extremes might be the sector-based multidisciplinary team, the intensive home treatment team for a given locality or the specialist multidisciplinary team delivering specific care programmes, e.g. eating disorders. For the purpose of this article, the clinical team is viewed as a multidisciplinary mental health clinical team with one consultant psychiatrist in the team.

For many of these teams the consultant psychiatrist may well be seen as the natural manager. For some teams there will undoubtedly be other professionals who will want to compete to be the manager. Even within smaller multidisciplinary teams it is quite legitimate for a non-medical person to be manager. However, for the clinical team to be 
effective it must be adequately resourced, operate within a clear corporate framework and enjoy effective leadership.

As manager of a clinical team, the consultant should be aware of what is happening in the external environment in order to make sense of the many demands made of the team.

\section{The management agenda}

Irrespective of which political party is in government technological advances, combined with greater consumer awareness and demand always outstripping resources, will be the driving forces for change in the health service. An enormous challenge will be faced by all managers involved in planning the delivery of new models of health care provision. Mental health professionals have, of course, been very successful in recent years in delivering new and innovative ways of working as the old psychiatric hospitals have been run down. The agenda for the next 10 years (Box 1), is likely to be even more complex and, as new styles of service delivery are developed, the clinical team will need strong leadership and sound management.

Box 1. Agenda for change

The vision of a primary care-led NHS

Health care purchasers demanding evidencebased services

A greater emphasis on prevention and health promotion

The deinstitutionalisation of much secondary care leading to fewer but more highly specialised general hospitals

Demands from service users for more choice in treatment models and a greater say in the planning and style of service delivery

More competition from the private and independent sectors

A demand from patients for more psychological treatment in preference to medication

The need for greater clarity on the division of responsibilities for providing secondary services and primary care services in mental health

Further blurring of professional boundaries in mental health and more shared territory for different care professionals
How then, will the management agenda impact on the working of the clinical team? It is clear that the drive towards a primary care-led NHS is already being felt by members of clinical teams. Since the introduction of fundholding, many GPs have examined alternative ways of dealing with patients who have mental health problems and they are considering treatments which are the most cost effective compared with the automatic referral of large numbers into the secondary care services. The number of GP employed primary practice counsellors has grown but many have no formal training. GPs are asking NHS Trusts to provide psychologists, CPNs, primary practice psychiatric nurses, and trained counsellors to deliver treatment in the primary care setting. This has led to many professionals becoming worried that some people with a mental illness might be receiving inappropriate treatment programmes and that resources are being diverted from the care of people with serious mental illness. In striving to meet the wishes of GPs, many clinical teams have been reluctant to filter referrals and reject inappropriate ones. They are conscious of the fact that GPs may well use their funds to buy alternative services if they are not getting what they want.

It also appears that many GPs perceive immense value from psychological treatments and fundholding has given them the ability to make greater use of clinical psychologists on a direct referral or even practice-based arrangement. This has led to claims from some quarters that clinical psychologists are setting themselves up in competition to psychiatrists. It is also leading to situations where clinical psychologists withdraw from the clinical team and concentrate on developing a more independent role in partnership with primary care colleagues. The Government's drive to a primary care-led NHS (NHS Executive, 1994) where there are more total fundholding GPs or, under a Labour Government where a system is introduced "which gives all GPs the opportunity to commission care" (Labour Party, 1995), will surely heighten these tensions and dilemmas. Whoever manages the clinical team will have to help mental health professionals to adapt and respond positively to the changes ahead. High quality leadership will be vital if a high standard of mental health care is to be maintained.

Whoever commissions mental health services in the future will demand care programmes and treatment methods based on sound research and measurable outcomes. The clinical team will be held accountable for the delivery of evidencebased services. This challenge is probably more difficult in the field of mental health than in any other branch of medicine. It will be for each 
professional group on the team to develop researchbased practice. The psychiatrist and psychologist have particular responsibilities here and whoever manages the clinical team has the unenviable job of ensuring the delivery of best practice while avoiding interprofessional power struggles. The clinical team manager needs to develop a culture of team working that recognises the centrality of the commissioner in the debate about clinical effectiveness. After the initial assessment, it should become the norm for the professional on the team with the lead clinical responsibility to negotiate with the GP a treatment timescale and set of likely clinical outcomes. This will require skills in negotiation, influencing and marketing, particularly for the team leader. Knowledge of research methodology and audit processes will be vital.

It is not only in mental health where services will move out of hospitals into new settings closer to the communities they serve. Technological changes will see the contraction of many general hospitals over the next few years as new patterns of health care develop. The polyclinic concept, with locally accessible day surgery facilities, diagnostic facilities and consultation services will lead to more general care being provided very close to patients' homes. More generic ways of working with district nurses, health visitors and hospital at home teams may have to be found. A fine balance will have to be struck between maintaining a specialist mental health identity and working alongside other healthcare workers in the community. Skills in team building and negotiation will be vital for the manager.

The user movement in this country was given added momentum by the 1991 NHS Reforms. Health Authorities were expected routinely to involve carers and users in the planning and development of services and to provide more choice in the models of care available locally. Rogers et al (1993) found that users are overwhelmingly supportive of hospital closures and see as disadvantageous the medicalisation of mental health problems and the tying of resources for mental health to hospital services. Some mental health professionals have concerns that the balance of influence is moving too far in favour of users and carers and that, as a consequence, the pattern of future provision will disadvantage many patients who need highly specialised in-patient care in settings which offer the most up-to-date treatment and diagnostic services. However, the move to a community centred mental health service at present seems unstoppable and the clinical team will have to respond to this re-orientation of care provision.
The management agenda for the NHS over the next decade is fairly predictable and coping with this agenda will be the business of all managers.

\section{Management and leadership}

In their recent article for this journal, Joyce \& Perkins (1996) took the view that psychiatrists should continue to have the major leadership role in mental health. There is no doubt that psychiatrists must continue to offer leadership in the development of mental health services and should play a pivotal part in its management. In doing so, psychiatrists must recognise that other professional groups will rightly have a major contribution to make to the development and management of services. Clinical psychology is an independent profession and psychiatrists must not seek to control the clinical activities of psychologists. Nurses similarly have a unique contribution to make to clinical care and as a profession nursing is becoming more autonomous. Initiatives are in progress to allow nurses to prescribe a limited range of medication and to move into more specialist areas like behavioural therapy. It is quite legitimate, but often controversial, for someone other than the consultant to manage the multidisciplinary clinical team. If the consultant does not manage the clinical team, this does not necessarily mean that they are giving up the leadership role. The consultant who does not manage the team may, as a consequence, become empowered to a surprising extent. As probably the most highly trained and experienced professional on the team, the consultant will most likely be sought out for advice, help and opinion to a greater extent than if he were the team manager.

What then do we mean by management and leadership in the context of the clinical team? The textbook definition talks about management in terms of the process of organising methods, materials, employees and other resources to achieve organisational goals. Sir Roy Griffiths saw the presence of a general management process in the NHS as providing staff leadership, bringing about a constant search for major change, obtaining cost improvements and securing the effective motivation of staff. Leadership is harder to define. There are numerous different schools of thought, the traditional one being that leaders are born, not made. The 'universal traits' theory suggests that leaders are people of commanding presence, decisive judgement, authoritative voices, good looks and boundless self-confidence! Another 
Box 2. Why doctors want to manage

A belief that the medical profession, because of its knowledge base, is best placed to make sensitive and sensible decisions about the use of scarce resources

A particular interest in the management sciences

A desire to shape services in a particular way and influence the overall culture of the organisation

A belief that management, with its control on resources, holds the key to delivering best clinical practice

A fascination with the political and managerial agenda

A belief that non-medical managers do not tackle the issues which matter

The need for a change in career direction to achieve a more stimulating and challenging role

school of thought, 'situational leadership', maintains that leaders are made, not born. Leaders here are seen to have no universal traits or behaviours, only situational ones. Leadership, to be effective, must in this model be appropriate to the situation. The consultant who aspires to lead the clinical team must spend some time considering and understanding the kind of leadership style appropriate to ensure success. As mentioned earlier, the team will need to operate responsively in a climate of considerable change created by the NHS reforms and the technological revolution. The traditional view of leadership assumes that people are powerless, lack personal vision and are unable to comprehend and handle the forces of change. Members of a clinical team in the NHS would not see themselves in this light. As suggested by Senge (1990) leaders are responsible for building organisations where people continually expand their capabilities to understand complexity, clarify vision and improve mental models. They are responsible for learning. The leader does not necessarily have the answer, but does have the quality to instil confidence in the team members so that they learn what is needed to deliver the desired outcomes. The consultant as leader must thrive on change. Change in this context is not about the structure of the NHS but the way the clinical team can improve its service to patients by constantly reviewing and changing its way of working. As stated by Peters (1988), the job of the leader is to "prepare people and organisations to deal with - to love, to develop affection for - change per se, as innovations are proposed, tested, rejected, modified and adopted".

The consultant who is successful in managing the clinical team will be the one who articulates a clear vision which team members have helped build. The vision will be communicated in a way which motivates colleagues and makes them feel they are part of something satisfying and meaningful.

\section{Why do consultants want to manage?}

The decision to move into management is not an easy one for most consultants. Doctors want to manage for many reasons (Box 2). Whatever the reason for entering management, there can be no doubt that consultants are well placed to give teams strong and effective leadership. The consultant understands the nature of the business better than most and has been trained to tackle difficult decisions, often of a life or death nature. The consultant understands the implications for patients of organisational and managerial decisions. The consultant is perceived by most other professional groups in the health service as enjoying legitimate power through high academic achievement and a clinical knowledge base greater than most others. The consultant has a strong case for being given the opportunity to manage the clinical team.

\section{The conflicts and dilemmas}

In preparing to take on any management role the consultant must address and come to terms with a whole range of conflicts and dilemmas.

Mascie-Taylor et al (1993) concluded that all doctors need help to explore, clarify and understand their values, both personal and professional, to enable them to take on board the managerial values required to operate effectively in the new Health Service. Consultants by the very nature of their work carry out management tasks. They manage their caseload, beds, junior doctors, day hospital places, etc. They usually participate in collegiate management through speciality divisions and they very often sit on task groups to consider and resolve particular problems. They believe that the doctor is clinically autonomous and responsible to the patient for providing the highest possible standard of individual care by applying the most up-to-date treatments within the bounds of medical knowledge. In the context of the NHS 
the belief sometimes extends to rule out any notion of rationing or prioritisation because of financial constraints. This is the extreme at one end of the spectrum of beliefs. Doctors do, of course, have to deliver clinical care within the resource limits at their disposal, but the doctor who has no real desire to become a manager might fight his speciality corner demanding more resources and developing new and often highly expensive techniques for which 'management' should find the money unless patients are to be expected to suffer.

There are many variations on the scale in terms of beliefs and values but it cannot be argued that the patient would not usually expect the doctor to fight for the highest quality and quantity of care possible. The manager, however, is concerned with the control of resources as well as the delivery of the best possible clinical outcomes. The manager will fight to secure the best financial allocation and shares the belief that the key goal of the NHS is to give patients the best deal possible. The manager does, nevertheless, have to face difficult decisions around rationing, cost control, stopping ineffective practice, prioritising between different care programmes and delivering the political agenda.

Some of these things may well run counter to the doctor's beliefs and values around the one to one relationship and accountability to the patient. This has always been a real issue for the consultant who takes on the management of a clinical directorate with responsibility for controlling a budget. Prior to the 1991 NHS reforms it was not a major issue for the manager of the clinical team but the increasing influence of purchasers, especially GPs, is creating new tensions and posing dilemmas. The consultant who takes on the management of the clinical team will have to face up to these dilemmas and the activity of preparing for management must involve a consideration of the conflicts that might arise and the strategies that may be needed to handle them.

\section{Management development}

As suggested in this paper, the process of preparing to take on a management role involves much more than simply attending a management course. Formal management training, however, should not be seen as optional. It is a very important component of the preparation process. It is a sad fact that many doctors who achieve consultant status have had little if any formal management training. The acquisition of managerial knowledge and skills should be a feature of both undergraduate and postgraduate education and those involved with curricula development should give the matter urgent attention. If we assume that the consultant who aspires to manage the clinical team has received no formal management training, then the importance of acquiring management knowledge must be recognised. The manager of the clinical team should ideally pursue a management development programme which contains factual information as well as providing skills-based training (Box 3).

Constructing the management development programme requires great care and attention. The consultant will find a vast number of different programmes available. However, the problem is that of tailoring the formal training to his individual needs. Advice should be sought from the Trust's Personnel Director and probably the Chief Executive. It would be a useful area to explore at the annual job plan meeting.

Training programmes specifically designed to meet the needs of doctors are readily available and are organised at regional level as well as by many universities and independent training agencies. These usually cover the basic areas listed below but are also useful in that they help the doctor explore the dilemmas of medicalism versus managerialism. They can also be particularly beneficial in offering opportunities for a group of doctors to discuss team building issues and group dynamics in a safe setting. This might be very appropriate when considering the difficulties surrounding the management of a multidisciplinary team where professional rivalry and control issues are often present. Exploring strategies and leadership styles appropriate to the management of teams might, for the consultant embarking on his first management job, be done more comfortably outside a multidisciplinary training session. While unidisciplinary training has its place, there are enormous gains to be made through training which involves doctors, other professional groups and general managers. Many courses are available which offer action learning and an opportunity to address ways of resolving real problems in the

Box 3. Topics for management training

The structure and organisation of the NHS The management agenda for the NHS Team building

Negotiating skills

Change management

Marketing

Presentation skills

Research methodologies 
Box 4. Management development checklist

Do I want a formal management qualification or is the acquisition of basic management skills and knowledge appropriate at the present time?

Does the course have credibility with colleagues who previously attended?

What is the calibre and reputation of the course tutor?

How much time can I devote to management training?

Is the course appropriate for meeting the criteria I have set in terms of skills and knowledge acquisition?

normal working environment. Perspectives on such problems from people in other sections of the NHS can be invaluable.

The consultant who takes on management of the clinical team would be well advised to explore ways of securing peer-group support. This can be achieved through action learning sets which to be successful require good facilitation and direction. Many management development programmes for doctors have a major learning set component and allow participants from different locations to share and resolve similar problems. Participants need to be open and honest about the problems they are encountering. It will usually be a comfort to know that others share similar problems which the group can address and devise strategies for resolving. The facilitator must be a highly skilled management trainer with detailed knowledge of the broad environment in which participants are working.

It is not the purpose of this paper to prescribe any particular programme of management development but to suggest that consultants look at their individual needs after reflecting on their personal career aspirations. Managing the clinical team will usually be the first major management challenge for the consultant and in choosing the right management development programme a checklist might be helpful (Box 4).

Completion of the management development programme is by no means the end of management training. It should be viewed in the context of continuing professional development in that management training must be on-going as the health service changes and adapts to face the different and growing demands placed upon it.

\section{Summary}

This paper has addressed the question of what consultants could reasonably do to prepare themselves for management of the clinical team. It suggests that preparing for a management role requires a detailed consideration of the social and political environment in which the NHS of today finds itself. The management agenda which has been set in response to environmental forces will undoubtedly impact on the working of all sectors of the NHS, including the clinical team. Consultants who manage the clinical team must face up to these challenges and acquire knowledge about the organisation of the NHS as well as management skills and techniques. Consultants are well placed to manage the clinical team but must recognise that by taking on this role they have a duty to their colleagues and their patients to equip themselves to do the job effectively.

\section{References}

Department of Health and Social Security (1983) NHS Management Inquiry. Letter - DA(83)88. London: DHSS.

- (1989) Working for Patients. Cm 555. London: DHSS.

Joyce, L. \& Perkins, R. (1996) A managerial perspective on consultant psychiatrists. Advances in Psychiatric Treatment, 2, 11-16.

Labour Party (1995) Renewing the NHS. Labour's Agenda for a Healthier Britain. London: Labour Party.

Mascie-Taylor, H., Pedler, M. J. \& Winkless, A. J. (1993) Doctors and Dilemmas. Leeds: NHS Executive.

NHS Executive (1994) Developing NHS Purchasing and GP Fundholding. Executive Letter EL(94)79. Leeds: NHSE.

Peters, T. (1988) Thriving on Chaos. London: Guild Publishing.

Rogers, A., Pilgrim, D. \& Lacey, R. (1993) Experiencing Psychiatry. Users' Views of Services. London: Macmillan in association with MIND publications.

Senge, P. M. (1990) The Fifth Discipline - The Art \& Practice of the Learning Organisation. New York: Doubleday. 